\title{
Translational Control by RNA-RNA Interaction Improved Computation of RNA-RNA Binding Thermodynamics
}

\author{
Ulrike Mückstein $^{1}{ }^{\star \star}$, Hakim Tafer ${ }^{1}$, Stephan H. Bernhart ${ }^{2}$, Maribel \\ Hernandez-Rosales ${ }^{2}$, Jörg Vogel ${ }^{3}$, Peter F. Stadler ${ }^{2,1,4,5}$, and Ivo L. Hofacker ${ }^{1}$ \\ 1 Institute for Theoretical Chemistry, University of Vienna, \\ Währingerstrasse 17, A-1090 Vienna, Austria \\ \{ulim, htafer, ivo\}@tbi.uvivie.ac.at, \\ http://www.tbi.univie.ac.at/ ivo/RNA/ \\ 2 Bioinformatics Group, Department of Computer Science, and Interdisciplinary \\ Center for Bioinformatics, University of Leipzig, Härtelstrasse 16-18, \\ D-04107 Leipzig, Germany \\ \{bstephan, maribel, studla\}@bioinf .uni-leipzig.de \\ 3 RNA Biology, Max Planck Institut für Infektionsbiologie, Charitéplatz 1, Campus \\ Charité Mitte, D-10117 Berlin, Germany \\ vogel@mpiib-berlin.mpg.de \\ 4 RNomics Group, Fraunhofer Institut for Cell Therapy and Immunology (IZI), \\ Deutscher Platz 5e, D-04103 Leipzig, Germany \\ 5 Santa Fe Institute, 1399 Hyde Park Rd., Santa Fe, NM 87501, USA
}

\begin{abstract}
The thermodynamics of RNA-RNA interaction consists of two components: the energy necessary to make a potential binding region accessible, i.e., unpaired, and the energy gained from the base pairing of the two interaction partners. We show here that both components can be efficiently computed using an improved variant of RNAup. The method is then applied to a set of bacterial small RNAs involved in translational control. In all cases of biologically active sRNA target interactions, the target sites predicted by RNAup is in perfect agreement with literature. In addition to prediction of target site location, RNAup can be also be used to determine the mode of sRNA action. Using information about target site location and the accessibility change resulting form sRNA binding we can discriminate between positive and negative regulators of translation.
\end{abstract}

\section{Introduction}

A series of high-throughput transcriptomics projects, among them ENCODE [1] and FANTOM [2] have demonstrated that mammalian genomes are pervasively transcribed, and that a large fraction of the transcripts does not code for proteins. Concurrently, small RNAs, in particular microRNAs and siRNAs have been identified as crucial regulators of gene expression, reviewed e.g. in [3].

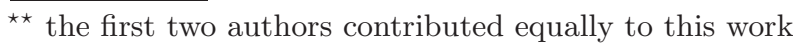




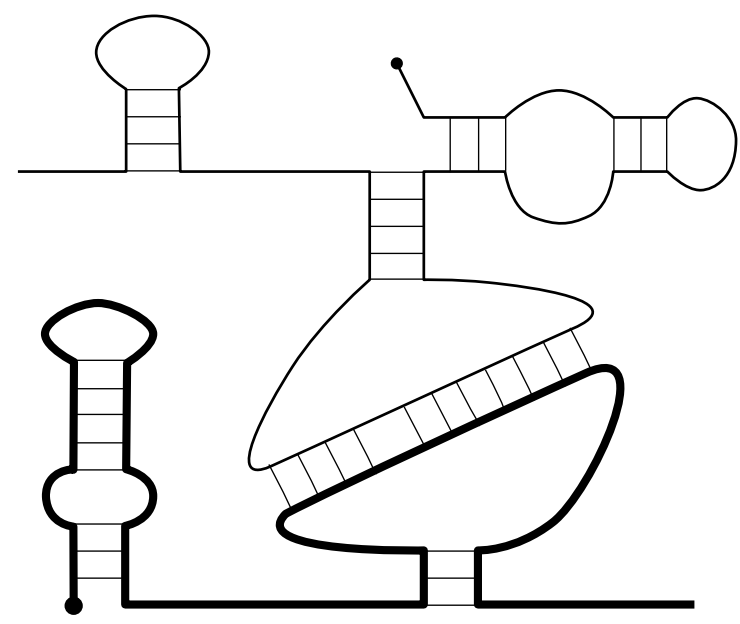

Fig. 1. Interaction between two RNAs of comparable length. Since each molecule forms intramolecular structures, the accessibility for an interactions differs along the molecule: Unstructured regions can easily take part in an interaction. Regions that are involved in an intramolecular structure, eg. the left hand side of the molecule drawn as a bold line, are not easily accessibly for intermolecular binding.

Genome-wide mapping of small ncRNAs [4] revealed novel classes of ncRNAs, implying that ncRNAs act by several, if not many, different mechanisms.

MicroRNAs, siRNAs, and snoRNAs require the direct interaction of ncRNAs and their target by means of base-pairing [5]. The same is true for many of the bacterial small RNAs that were discovered during the last decade, see e.g. [6]. Computational evidence [7] suggests, furthermore, that a significant fraction of RNA candidates with evolutionary conserved RNAs [8] binds to mRNAs.

These observations have triggered increasing interest in methods to predict "targets" via the evaluation of RNA-RNA interactions. For microRNAs, the available tools are almost too numerous to list (see $[9,10]$ for recent reviews), targetRNA [11] is frequently used for bacteria, and a specific heuristic for orphan snoRNAs was presented recently [12]. In the simplest case, only the base pairing between the two interacting partners is taken into account [13-16,11]. In most cases, however, RNA-RNA interaction does not cover the entire target. This is maybe most evident in the case of short siRNAs or miRNAs targeting long mRNAs. It becomes necessary in such cases, to explicitly consider the structure of the target. In [17], anti-sense target are predicted as unpaired regions on the target molecules. For siRNA and microRNA is was shown that the accessibility of the target site correlates directly with the efficiency of cleavage $[18,19]$.

Instead of treating the target independent of its binding partner, it seems more appealing to compute the structure of the interaction complex. Just as the folding problem with pseudoknots [20], finding the energetically optimal interaction structure is NP-complete [21]. It is, however not even desirable to solve 
the general "RIP" problem, because too highly entangled structures typically are not formed in nature. Practical approaches there restrict the set of interaction structures that are searched. So far, four classes of structures have been investigated in some detail:

1. Only base-pairs between the interacting RNAs are considered, no base pairs are allowed within each structure. As argued above, disregarding the internal structure of the interaction partners is too crude an approximation

2. Interactions between the two molecules are restricted to the external bases of the two partners. Such structures can be computed by means of a straightforward generalization of the usual pseudoknot-free folding algorithm [22, 23]. This class of structures, however, is still too restrictives as it rules out frequent motifs such as kissing-hairpins [24].

3. The other extreme is to consider all "tangle-free" interaction structures. This leads to a rather expensive algorithm with a runtime $\mathcal{O}\left(m^{3} \cdot n^{3}\right)$ and quartic memory consumption $[25,21,26,27]$, which is prohibitive for many largescale applications. Another problem is that the interaction structures contain many types of complex loops for which energy parameters are unknown.

4. The RNAup approach [28] restricts the region of interaction to a single interval on each of the interaction partners, while arbitrary pseudoknot free structures are allowed elsewhere, Fig. 1. This model is sufficient for most but not all known RNA-RNA interactions. For example, the OxyS-fhlA interaction [29] contains two separate kissing complexes and can therefore not be predicted using RNAup. Most bacterial sRNAs however show one well defined interaction with a typical interaction length from 9 bp up to 60 bp and variable degrees of complementarity between ncRNAs and target sequence [30, 31]. In [28], only the target molecule was assumed to be structured, while the ncRNA partner was assumed to be a miRNA or siRNA without internal structure. Here we will drop this restriction.

Instead of directly computing the interaction structure, RNAup decomposes the problem into three steps: For each subsequence (with bounds $i$ and $j$ ) of an RNA, we compute the probability $P[i, j]$ that it is unpaired. This probability is equivalent to the free energy of making the binding regions accessible. The optimal interaction structure is than computed by assessing all possible combinations of binding sites of both partners.

This conceptual decomposition of RNA/RNA binding into an unfolding and an interaction contribution has most recently been utilized by several groups. Long et al. [32] developed a model for modeling the interaction between a miRNA and a target as a two-step hybridization reaction: nucleation at an accessible target site, followed by hybrid elongation to disrupt local target secondary structure and form the complete miRNA-target duplex. Lu \& Mathews [33] predicted the cost of opening base pairs in the mRNA for hybridization to siRNA by calculating the structure once without constraints and then once with the constraint that the nucleotides in the hybridization site are forced single-stranded. Kertesz et al. [19] devised a parameter-free model for microRNA-target interaction that computes the difference between the free energy gained from the formation of 
the microRNA-target duplex and the energetic cost of unpairing the target to make it accessible to the microRNA.

In the following section we first describe an algorithmic improvement in the computation of $P[i, j]$ that leads to a significant speed-up, then we generalize RNAup to accommodating two partners with internal secondary structures. These improvements put us into the position to study translational control by bacterial sRNA.

\section{Algorithm}

RNAup calculates the energetics of RNA-RNA interactions in a step-wise process. The free energy of binding $\Delta G$ consists of the "breaking energies" $\Delta G_{u}$ that are necessary to transform the binding site in each molecule into an accessible conformation and a contribution $\Delta G_{h}$ that describes the energy gain due to hybridization:

$$
\Delta G=\Delta G_{u}^{A}+\Delta G_{u}^{B}+\Delta G_{h},
$$

where $A$ and $B$ denote the two interacting molecules. In principle, Equ. 1 has to be evaluated for every possible combination of interacting regions in molecule $A$ and $B$. In practice, our algorithm first computes the accessibilities $\Delta G_{u}$ for all regions up to a maximal size $w$, and then combines these regions to compute the hybridization energies $\Delta G_{h}$.

In order to compute free energies of binding we cannot rely on finding a single optimal structure only. Instead, we have to compute the partition functions associated with these three free energy terms. This can be done by (suitably modified) variants of the algorithm introduced by McCaskill's algorithm [34] and and implemented in the Vienna RNA package [35]. Recall that the equilibrium partition function is defined as

$$
Z=\sum_{S} \exp (-\beta F(S)),
$$

where $F(S)$ is the free energy of a secondary structure $S$, and $\beta=1 /(R T)$ stands for the inverse of the temperature times Boltzmann's constant (here expressed as the gas constant, i.e., for energies per mol). Note that individual secondary structures are assigned temperature dependent free energies with entropic contributions arising from the ensemble of microscopic conformations that are assigned to a single secondary structure as macrostate. Energy parameters used here are taken from [36]

\subsection{Calculation of Accessibility}

Taken together, partition functions for subsequences contain the information necessary to compute the frequency of structural motifs, in the simplest case individual unpaired bases of base pairs [34]. Here, we are interested in the probability $P_{u}[i, j]$ that the sequence interval $[i, j]$ is unpaired, which is equivalent 
to the energy $\Delta G_{u}[i, j]=-R T \ln \left(P_{u}[i, j]\right)$ necessary to make the subsequence from $i$ to $j$ single-stranded.

An unpaired interval $[i, j]$ is either "exterior" , i.e. not enclosed by a basepair, or there exists an enclosing base pair $(p, q)$ such that $p<i<j<q$ and there is no other pair $(s, t)$ such that $p<s<i<j<t<q$. We can therefore express $P_{u}[i, j]$ in terms of restricted partition functions for these two cases:

$$
P_{u}[i, j]=\frac{Z(1, i-1) Z(j+1, n)+\sum_{p<i} \sum_{j<q} \hat{Z}(p, q) Z_{p q}[i, j]}{Z(1, n)}
$$

where $\hat{Z}(p, q)$ is the partition function outside base pair $p, q$, and $Z_{p q}[i, j]$ the partition function inside a basepair $p, q$ given that the interval $[i, j]$ is unpaired. We introduce here an improved recursion for $\hat{Z}(p, q) Z_{p q}[i, j]$ that reduces the CPU requirements of the previous implementation of RNAup [37] from $\mathcal{O}\left(n^{3} \cdot w\right)$ to $\mathcal{O}\left(n^{3}\right)$, where $n$ is the length of the sequence and $w$ is the maximal size of the unstructured region $[i, j]$. This results in a substantial performance gain that considerably facilitates large-scale applications.

As in [37], we start from the observation that $Z_{p q}[i, j]$ consists of three contributions, of which the summation of all multi-loop energies is the most complex one. This multi-loop part is itself again split into three parts, dependings on whether the unpaired region is to the left or to the right of all components of a multi-loop or in between them, Fig. 2:

$$
\begin{aligned}
Z^{\text {mult }}[i, j] & =\sum_{p<i<j<q} \hat{Z}(p, q) \times \\
& (\underbrace{\underbrace{M 2}(p+1, i-1) e^{-\beta c(q-i)}}_{\text {left }}+\underbrace{Z^{M 2}(j+1, q-1) e^{-\beta c(j-p)}}_{\text {right }} \\
& +\underbrace{Z^{M}(p+1, i-1) e^{-\beta c(j-i+1)} Z^{M}(j+1, q-1)}_{\text {in-between }})
\end{aligned}
$$

The crucial improvement is obtained by replacing the double sum in Eq. 3 by two separate summation steps. For the last, "in-between", summand we use the auxiliary variables

$$
Z^{M M}(q)[i]=\sum_{1 \leq p<i} \hat{Z}(p q) Z^{M}(p+1, i-1)
$$

for $Z_{l}^{M}(q)[i]$ where the unpaired region $[i, j]$ lies to the left of all multi-loop components, we introduce

$$
Z_{l}^{M}(q)[i]=\sum_{1 \leq p<i} \hat{Z}(p, q) Z^{M 2}(p+1, i-1) e^{-\beta c(q-i)}
$$




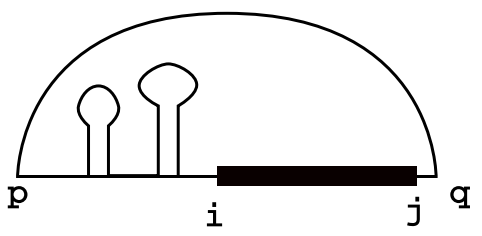

A

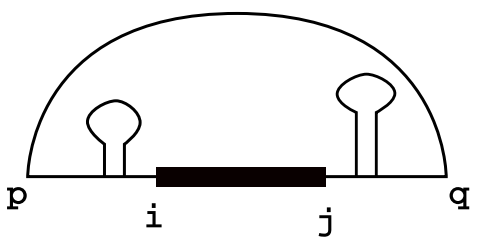

B

Fig. 2. Decomposition for calculating multiloop contributions: Base pair $[p, q]$ that includes the unpaired region $[i, j]$ is drawn as an arc connecting bases $p$ and $q$. The unpaired region $[i, j]$ is drawn as a bold black line. In the the one-sided multiloop case (A) a structured region containing at least two structure components is on one side of the unpaired region. In case (B) the unpaired region $[i, j]$ is between two structured regions. In case (B) we have to take care to make a unique decomposition of the multiloop into a 3' part that contains exactly one component and a 5' part with at least one component.

and an analgous term is used for the "right" contribution. Computing these values costs $\mathcal{O}\left(n^{3}\right)$. By using them, we can compute

$$
\begin{aligned}
Z^{\text {mult }}[i, j] & =\sum_{j<q} Z^{M M}(q)[i] e^{-\beta c(j-i+1)} \\
& +\sum_{p<i} Z_{r}^{M}(p)[j] \\
& +\sum_{j<q} Z^{M}(j+1, q-1)+Z_{l}^{M}(q)[i]
\end{aligned}
$$

in $\mathcal{O}\left(n^{2} \cdot w\right)$ time, i.e., the entire algorithm is $\mathcal{O}\left(n^{3}\right)$. The computations for hairpin and interior loop contributions are handled in the same way.

In comparison to McCaskill's partition function algorithm, RNAup needs to store five additional matrices $\left(Z^{M 2}, Z^{M M}, Z_{l}, Z_{r}\right.$ and one additional matrix for the interior loop case). Hence we buy the speed-up by $\mathcal{O}(w)$ by increasing the memory requirements by only about a factor of 2 .

\subsection{Free Energy of Interaction}

In [37] we used $P_{u}[i, j]$ only for the (long) target mRNA, assuming that the siRNA or miRNA is unstructured due to its short length. This approximation cannot be justified for most bacterial small RNAs, however. Hence, we extended RNAup to take the secondary structure of both interacting molecules into account.

Suppose the interaction region covers the intervals $\left[i^{*}, j^{*}\right]$ and $[i, j]$ in the two RNAs. As in RNAhybrid and related programs, we allow interior loops and bulges in the interaction region. The partition function over all these binding 
conformations is obtained by the following recursion:

$$
Z^{I}\left[i, j, i^{*}, j^{*}\right]=\sum_{\substack{i<k<j \\ i^{*}>k^{*}>j^{*}}} Z^{I}\left[i, k, i^{*}, k^{*}\right] e^{-\beta I\left(k, k^{*} ; j, j^{*}\right)} .
$$

where $I\left(k, k^{*} ; j, j^{*}\right)$ is the energy contribution for the interior loop delimited by the base pairs $\left(k, k^{*}\right)$ and $\left(j, j^{*}\right)$.

As we want to avoid having to keep track of a four dimensional array, we compute the partition function $Z^{*}[i, j]$ over all structures where region $[i, j]$ in the longer molecule is involved in the interaction. While doing this, we keep track of the region where $Z^{I}\left[i, j, i^{*}, j^{*}\right.$ is maximal. The recursion for the calculation of $Z^{*}[i, j]$ is shown in Eq 9 .

$$
Z^{*}[i, j]=P_{u}^{A}[i, j] \sum_{i^{*}>j^{*}} P_{u}^{B}\left[i^{*}, j^{*}\right] Z^{I}\left[i, j, i^{*}, j^{*}\right] .
$$

From $Z^{*}[i, j]$ we can readily compute $\Delta G[i j]$, the free energy of binding given the binding site is in region $[i, j]$. For visual inspection, $\Delta G[i j]$ can be reduced to the optimal free energy of binding at a given position $i$, see Eq 10 . The memory requirement for these steps is $\mathcal{O}\left(n \cdot w^{3}\right)$, the required CPU time scales as $\mathcal{O}\left(n \cdot w^{5}\right)$, which, at least for long target RNAs, is dominated by the first step, i.e., the computation of the $P_{u}[i, j]$.

$$
\begin{aligned}
\Delta G[i, j] & =-R T \ln Z^{*}[i, j] . \\
\Delta G[i] & =\min _{k \leq i \leq l}\{\Delta G[k, l]\} .
\end{aligned}
$$

The positional free energy, $\Delta G_{i}$, referring to position $i$ in the target molecule, is written to a file suitable for plotting. For the region with maximal $Z^{I}\left[i, j, i^{*}, j^{*}\right.$, we use RNAduplex to print out the optimal interaction structure.

\section{Results}

In order to test the reliability of RNAup for target sRNAs prediction, we used a dataset consisting of 9 small regulatory RNAs from E.Coli, their 9 reported mRNA targets and the fold-change in protein concentration induced by all 81 possible mRNA-ncRNA interactions [30]. Among those interactions, 8 targets were downregulated, 2 were upregulated, and no or only marginal changes were detected for the others (see Table 1). Downregulation usually occurs when the hybridisation of the ncRNA with its cognate mRNA blocks the ribosome entry sites on the target (for a review see [38]). In contrast, upregulation typically disrupts intrinsic inhibitory structures that sequester the ribosome binding site and/or the start codon [39-41]. In many cases the sRNA-mRNA interactions are assisted by the RNA chaperone protein $H f q$ [42].

Target prediction was performed with the mRNA constructs (117-689 nts) described in [30] and the full length sRNAs (69-220 nts). The mRNA constructs 
Table 1. Binding site summary for the 10 functional interactions published by Urban et.al [30]. The number in parenthesis represents the quantity of non-functional interactions involving the same ncRNA with a higher interaction energy than the functional hybrid. Column Position gives the binding position relative to the start codon computed with RNAup.Column Position lit. gives the binding position found in the literature.

\begin{tabular}{llllll}
\hline mRNA sRNA regulation & $\Delta \Delta G$ & Position & Pos.lit. & cite \\
\hline RyhB sodB & - & -11.50 & $-18,+4$ & $-4,+5$ & {$[43]$} \\
DsrA hns & - & -14.60 & $-10,+11$ & $+7,+19$ & {$[44]$} \\
MicA ompA & - & -13.60 & $-21,-6$ & $-21,-6$ & {$[45]$} \\
MicC ompC & - & -15.80 & $-30,-15$ & $-30,-15$ & {$[46]$} \\
MicF ompF & - & -17.80 & $-11,+9$ & $-11,+10$ & {$[46]$} \\
Spot42 galK & - & -17.00 & $-18,+30$ & $-19,+21$ & {$[47]$} \\
SgrS ptsG & - & -17.33 & $-28,-10$ & $-28,+4$ & {$[48]$} \\
GcvB dppA & - & -17.30 & $-30,-7$ & $-31,-14$ & {$[31]^{a}$} \\
DsrA rpoS & + & -14.52 & $-126,-97$ & $-119,-97$ & {$[40]$} \\
RprA rpoS & + & -15.90 & $-134,-94$ & $-117,-94$ & {$[40]$} \\
\hline${ }_{\text {GcvB } / \text { dppA interaction was studied in Salmonella enterica serovar Typhimurium not in E.coli. }}$
\end{tabular}

included a long 5'UTR sequence (57-565 nts) and a comparably short fragment of the CDS (35-139 nts). Both the hybridisation energy and the target site position was computed with RNAup for all sRNA-mRNA combinations.

For each sRNA we tested which of the mRNA construct was predicted to bind more strongly. To our satisfaction the most favorable binding energy for each sRNAs was found for their cognate targets (see Table 1).

Furthermore, we checked whether target sites returned by RNAup were in agreement with the experimentally determined binding sites from [30]. In all cases the predicted binding sites overlapped with experimental ones, in 9 out 10 cases the agreement was almost perfect.

It is of interest not only to predict interaction sites, but also the regulatory effect the interaction might have. Since the most common mechanism of translational control is to influence ribosome binding at the shine-dalgarno (SD) sequence, we checked the position and structural effects of the predicted interactions.

For each of the 8 interactions that result in downregulation, we found the binding site to be at or close to the shine-dalgarno sequence. This type of inhibition can thus be predicted by comparing RNAup predictions with sequence features that are easy to recognize in bacterial genomic sequences.

Our data set contains only two examples of upregulation, namely binding of DsrA and RprA to rpoS. In both cases, binding leads to the disruption of a helix which normally sequesters the shine-dalgarno sequence as well as the start codon. We remark that this is an example of the modifier RNA mechanism that was proposed in $[49,50]$. 


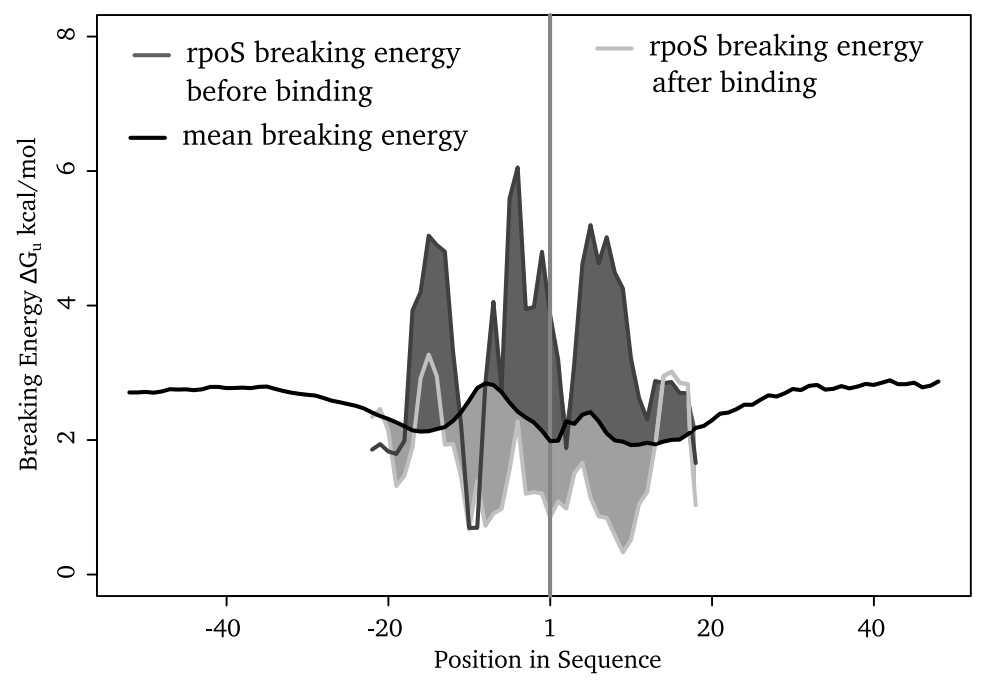

Fig. 3. Opening energy, $\Delta G_{u}$ plotted versus sequence position for the interaction of DsrA with textitrpoS. The vertical gray line marks the position of the start codon. The black line represents the average breaking energy for all E. Coli mRNAs. The dark gray line represents the opening energy of unbound $r p o S$, the light gray line the opening energy after binding $D s r A$. Unbound $r p o S$ is less accessible than average (dark gray area), while bound $r p o S$ is more accessible than average (light gray area).

To assess the ability of RNAup to predict upregulating interactions we first compared the accessibility of the region around the start codon of all 9 mRNAs, with the mean accessibility of all 4463 genes in the E.Coli genome. Mean accessibility was computed for regions of 401 nts, centered at the start codon. For comparability we used, the same $401 \mathrm{nts}$ regions of our 9 target genes rather than the constructs used above. The accesssibilities and corresponding opening eneries were computed with RNAup for unpaired regions of length 4 .

With a local opening energy of $4.51 \mathrm{kcal} / \mathrm{mol} \mathrm{rpoS}$ is the most inaccessible transcripts among the 9 presented here. Genome-wide only $8.8 \%$ of the transcripts have a less accessible start codon than rpoS. In contrast, the eight downregulated transcripts showed a higher than average $(2.23 \mathrm{kcal} / \mathrm{mol})$ accessibility, ranging from $0.30 \mathrm{kcal} / \mathrm{mol}$ for ompA to a maximum of $1.27 \mathrm{kcal} / \mathrm{mol}$ for ryhB.

After binding $D s r A$, the accessibility of the rpoS start codon changes dramatically. With only $1.40 \mathrm{kcal} / \mathrm{mol}$, bound rpoS is much more accessible than the average transcript and belongs to the $33 \%$ most accessible genes, see fig. 3 . The same effect is seen upon binding with $\operatorname{Rpr} A$, with a local accessibility after binding of $1.90 \mathrm{kcal} / \mathrm{mol}$. Technically, accessibilities after binding can be computed easily by adding the constraint that nucleotides in the binding site remain single stranded. 


\section{Conclusion}

Translational control by sRNAs is an important regulatory function throughout all bacteria. In contrast to e.g. micro RNAs, these regulatory RNAs are mostly structured. We have improved RNAup to take both target and sRNA structure into account. As we have also increased the speed of RNAup, it is now suitable for the computational identification of mRNA targets of bacterial sRNAs.

Furthermore, we find that RNAup can be used to predict the regulatory effect of sRNA binding by investigating the location of the binding site and the structural changes induced by binding in the vicinity of the start codon of the mRNA. A predicted binding close to the start codon or the shine-dalgarno sequence is a clear indicator for downregulation. While results look promising for upregulation, too, a bigger data set is needed to confirm that RNAup can accurately predict that, too.

Our algorithm captures the most common types of interaction between regulatory RNAs and their targets, even though more complicated types of interactions, such H/ACA snoRNA with their target rRNAs or OxyS-fhlA, are neglected. The speed of RNAup is clearly sufficient for genome wide searches for sRNA-mRNA interactions in bacteria. In principle, the approach is equally applicable to interaction search in higher organisms. However, the larger genome size and longer UTR regions pose challenges both in terms of computation time and false positives.

\section{Acknowledgments}

This work has been funded, in part, by the Austrian GEN-AU projects bioinformatics integration network and non-coding RNA, the FP-6 EMBIO project, the Deutsche Forschungsgemeinschaft Proj No STA 850/7-1 as part of SPP-1258 "Sensory and Regulatory RNAs in Prokaryotes" and Siemens. 


\section{References}

1. The ENCODE Project Consortium: Identification and analysis of functional elements in $1 \%$ of the human genome by the ENCODE pilot project. Nature 447 (2007) 799-816

2. Maeda, N., Kasukawa, T., Oyama, R., Gough, J., Frith, M., Engström, P.G., Lenhard, B., Aturaliya, R.N., Batalov, S., Beisel, K.W., Bult, C.J., Fletcher, C.F., Forrest, A.R., Furuno, M., Hill, D., Itoh, M., Kanamori-Katayama, M., Katayama, S., Katoh, M., Kawashima, T., Quackenbush, J., Ravasi, T., Ring, B.Z., Shibata, K., Sugiura, K., Takenaka, Y., Teasdale, R.D., Wells, C.A., Zhu, Y., Kai, C., Kawai, J., Hume, D.A., Carninci, P., Hayashizaki, Y.: Transcript annotation in FANTOM3: Mouse gene catalog based on physical cdnas. PLoS Genetics 2 (2006) e62 doi:10.1371/journal.pgen.0020062.

3. Mattick, J.S., Makunin, I.V.: Non-coding RNA. Hum Mol Genet. 15 (2006) R1729

4. Kapranov, P., Cheng, J., Dike, S., Nix, D., Duttagupta, R., Willingham, A.T., Stadler, P.F., Hertel, J., Hackermüller, J., Hofacker, I.L., Bell, I., Cheung, E., Drenkow, J., Dumais, E., Patel, S., Helt, G., Madhavan, G., Piccolboni, A., Sementchenko, V., Tammana, H., Gingeras, T.R.: RNA maps reveal new RNA classes and a possible function for pervasive transcription. Science 316 (2007) 1484-1488

5. Schubert, S., Grnweller, A., Erdmann, V.A., Kurreck, J.: Local RNA target structure influences siRNA efficacy: systematic analysis of intentionally designed binding regions. J Mol Biol 348(4) (May 2005) 883-893

6. Vogel, J., Wagner, E.G.: Target identification of small noncoding RNAs in bacteria. Curr Opin Microbiol. 10 (2007) 262-270

7. The Athanasius F. Bompfünewerer RNA Consortium:, Backofen, R., Flamm, C., Fried, C., Fritzsch, G., Hackermüller, J., Hertel, J., Hofacker, I.L., Missal, K., Mosig, Axel Prohaska, S.J., Rose, D., Stadler, P.F., Tanzer, A., Washietl, S., Sebastian, W.: RNAs everywhere: Genome-wide annotation of structured RNAs. J. Exp. Zool. B: Mol. Dev. Evol. 308B (2007) 1-25

8. Washietl, S., Hofacker, I.L., Lukasser, M., Hüttenhofer, A., Stadler, P.F.: Mapping of conserved RNA secondary structures predicts thousands of functional noncoding RNAs in the human genome. Nature Biotech. 23 (2005) 1383-1390

9. Doran, J., Strauss, W.M.: Bio-informatic trends for the determination of miRNAtarget interactions in mammals. DNA Cell Biol 26 (2007) 353-360

10. Maziére, P., Enright, A.J.: Prediction of microRNA targets. Drug Discov Today 12 (2007) 452-458

11. Tjaden, B., Goodwin, S.S., Opdyke, J.A., Guillier, M., Fu, D.X., Gottesman, S., Storz, G.: Target prediction for small, noncoding RNAs in bacteria. Nucleic Acids Res. 34 (2006) 2791-2802

12. Bazeley, P.S., Shepelev, V., Talebizadeh, Z., Butler, M.G., Fedorova, L., Filatov, V., Fedorov, A.: snoTARGET shows that human orphan snoRNA targets locate close to alternative splice junctions. Gene 408 (2008) 172-179

13. Rehmsmeier, M., Steffen, P., Hochsmann, M., Giegerich, R.: Fast and effective prediction of microRNA/target duplexes. RNA 10(10) (Oct 2004) 1507-1517

14. Zuker, M.: Mfold web server for nucleic acid folding and hybridization prediction. Nucleic Acids Res 31(13) (Jul 2003) 3406-3415

15. Dimitrov, R.A., Zuker, M.: Prediction of hybridization and melting for doublestranded nucleic acids. Biophys J 87(1) (Jul 2004) 215-226 
16. Hodas, N.O., Aalberts, D.P.: Efficient computation of optimal oligo-RNA binding. Nucleic Acids Res 32(22) (2004) 6636-6642

17. Ding, Y., Lawrence, C.E.: Statistical prediction of singletranded regions in RNA secondary structure and application to predicting effective antisense target sites and beyond. Nucl. Acids Res. 29 (2001) 1034-1046

18. Ameres, S.L., Martinez, J., Schroeder, R.: Molecular basis for target RNA recognition and cleavage by human RISC. Cell 130(1) (Jul 2007) 101-112

19. Kertesz, M., Iovino, N., Unnerstall, U., Gaul, U., Segal, E.: The role of site accessibility in microRNA target recognition. Nat Genet 39(10) (Oct 2007) 1278-1284

20. Akutsu, T.: Dynamic programming algorithms for RNA secondary structure with pseudoknots. Discrete Applied Mathematics 104 (2000) 45-62

21. Alkan, C., Karakoç, E., Nadeau, J.H., Sahinalp, S.C., Zhang, K.: RNARNA interaction prediction and antisense RNA target search. J. Comp. Biol. 13 (2006) $267-282$

22. Andronescu, M., Zhang, Z.C., Condon, A.: Secondary structure prediction of interacting RNA molecules. J Mol Biol 345(5) (Feb 2005) 987-1001

23. Bernhart, S.H., Tafer, H., Mückstein, U., Flamm, C., Stadler, P.F., Hofacker, I.L.: Partition function and base pairing probabilities of RNA heterodimers. Algorithms Mol. Biol. 1 (2006) 3 [epub]

24. Wagner, E.G.H., Simons, R.W.: Antisense RNA control in bacteria, phage, and plasmids. Annu. Rev. Microbiol. 48 (1994) 713742

25. Pervouchine, D.D.: IRIS: Intermolecular RNA interaction search. Proc. Genome Informatics 15 (2004) 92-101

26. Aksay, C., Salari, R., Karakoc, E., Alkan, C., Sahinalp, S.C.: taveRNA: a web suite for RNA algorithms and applications. Nucleic Acids Res 35(Web Server issue) (Jul 2007) W325-W329

27. Kato, Y., Akutsu, T., Seki, H.: A grammatical approach to RNA-RNA interaction prediction. AIP Conf. Proc. 952 (2007) 197-206 CMLS '07: 2007 International Symposium on Computational Models of Life Sciences.

28. Mückstein, U., Tafer, H., Hackermüller, J., Bernhard, S.B., Stadler, P.F., Hofacker, I.L.: Thermodynamics of RNA-RNA binding. Bioinformatics 22 (2006) 1177-1182

29. Argamana, L., Altuvia, S.: fhla repression by oxys rna: kissing complex formation at two sites results in a stable antisense-target rna complex. J Mol Biol. 300(5) (2000) 1101-12

30. Urban, J.H., Vogel, J.: Translational control and target recognition by Escherichia coli small RNAs in vivo. Nucleic Acids Res 35(3) (2007) 1018-1037

31. Sharma, C.M., Darfeuille, F., Plantinga, T.H., Vogel, J.: A small RNA regulates multiple ABC transporter mRNAs by targeting C/A-rich elements inside and upstream of ribosome-binding sites. Genes Dev 21(21) (Nov 2007) 2804-2817

32. Long, D., Chan, C.Y., Ding, Y.: Analysis of microRNA-target interactions by a target structure based hybridization model. Pac Symp Biocomput (2008) 64-74

33. Lu, Z.J., Mathews, D.H.: Efficient siRNA selection using hybridization thermodynamics. Nucleic Acids Res 36(2) (Feb 2008) 640-647

34. McCaskill, J.S.: The equilibrium partition function and base pair binding probabilities for RNA secondary structure. Biopolymers 29(6-7) (1990) 1105-1119

35. Hofacker, I., Fontana, W., Stadler, P., Bonhoeffer, S., Tacker, M., Schuster, P.: Fast folding and comparison of RNA secondary structures. Monatsh. Chem. 125 (1994) 167-188

36. Mathews, D.H., Sabina, J., Zuker, M., Turner, D.H.: Expanded sequence dependence of thermodynamic parameters improves prediction of RNA secondary structure. J Mol Biol 288(5) (May 1999) 911-940 
37. Mckstein, U., Tafer, H., Hackermller, J., Bernhart, S.H., Stadler, P.F., Hofacker, I.L.: Thermodynamics of RNA-RNA binding. Bioinformatics 22(10) (May 2006) $1177-1182$

38. Gottesman, S.: Micros for microbes: non-coding regulatory rnas in bacteria. Trends Genet 21(7) (Jul 2005) 399-404

39. Majdalani, N., Cunning, C., Sledjeski, D., Elliott, T., Gottesman, S.: Dsra rna regulates translation of rpos message by an anti-antisense mechanism, independent of its action as an antisilencer of transcription. Proc Natl Acad Sci U S A 95(21) (Oct 1998) 12462-12467

40. Majdalani, N., Hernandez, D., Gottesman, S.: Regulation and mode of action of the second small RNA activator of RpoS translation, RprA. Mol Microbiol 46(3) (Nov 2002) 813-826

41. Prévost, K., Salvail, H., Desnoyers, G., Jacques, J.F., Phaneuf, E., Massé, E.: The small rna ryhb activates the translation of shia mrna encoding a permease of shikimate, a compound involved in siderophore synthesis. Mol Microbiol 64(5) (Jun 2007) 1260-1273

42. Valentin-Hansen, P., Eriksen, M., Udesen, C.: The bacterial sm-like protein hfq: a key player in rna transactions. Mol Microbiol 51(6) (Mar 2004) 1525-1533

43. Geissmann, T.A., Touati, D.: Hfq, a new chaperoning role: binding to messenger RNA determines access for small RNA regulator. EMBO J 23(2) (Jan 2004) 396405

44. Lease, R.A., Cusick, M.E., Belfort, M.: Riboregulation in Escherichia coli: DsrA RNA acts by RNA:RNA interactions at multiple loci. Proc Natl Acad Sci U S A 95(21) (Oct 1998) 12456-12461

45. Rasmussen, A.A., Eriksen, M., Gilany, K., Udesen, C., Franch, T., Petersen, C., Valentin-Hansen, P.: Regulation of ompA mRNA stability: the role of a small regulatory RNA in growth phase-dependent control. Mol Microbiol 58(5) (Dec 2005) 1421-1429

46. Chen, S., Zhang, A., Blyn, L.B., Storz, G.: MicC, a second small-RNA regulator of Omp protein expression in Escherichia coli. J Bacteriol 186(20) (Oct 2004) 6689-6697

47. Mller, T., Franch, T., Udesen, C., Gerdes, K., Valentin-Hansen, P.: Spot 42 RNA mediates discoordinate expression of the E. coli galactose operon. Genes Dev 16(13) (Jul 2002) 1696-1706

48. Kawamoto, H., Koide, Y., Morita, T., Aiba, H.: Base-pairing requirement for RNA silencing by a bacterial small RNA and acceleration of duplex formation by Hfq. Mol Microbiol 61(4) (Aug 2006) 1013-1022

49. Meisner, N.C., Hackermüller, J., Uhl, V., Aszódi, A., Jaritz, M., Auer, M.: mRNA openers and closers: A methodology to modulate AU-rich element controlled mRNA stability by a molecular switch in mRNA conformation. Chembiochem. 5 (2004) 1432-1447

50. Hackermüller, J., Meisner, N.C., Auer, M., Jaritz, M., Stadler, P.F.: The effect of RNA secondary structures on RNA-ligand binding and the modifier RNA mechanism: A quantitative model. Gene 345 (2005) 3-12 